\title{
Erratum to: Indivisibility of Electron Bubbles in Helium
}

\author{
V. Elser ${ }^{1}$
}

Erratum to: Journal of Low Temperature Physics, Vol. 123, Nos. 1/2, 2001 DOI 10.1023/A:1017534129860

I thank Mateo et al. [1] for pointing out an error in the original article where Eq. (3) was substituted into Eq. (8). The corrected Eq. (9) is as follows:

$$
\frac{a}{R_{2}}=\left(\frac{27}{8 \pi^{4} \mu} \cdot \frac{m_{e}}{\rho R_{0}^{3}} \cdot \frac{R_{2}}{R_{0}}\right)^{1 / 9}=0.13
$$

The resulting orifice diameter is $2 a=4.2 \AA$. This does not alter the main conclusion that the adiabatic approximation breaks down well before the double bubble has completely fissioned. If anything, the decrease in $a$ improves the reliability of the approximations made.

\section{Reference}

1. D. Mateo, M. Pi, M. Barranco, Phys. Rev. B 81, 174510 (2010)

The online version of the original article can be found under doi:10.1023/A:1017534129860.

$\otimes$ V. Elser

ve10@cornell.edu

1 Department of Physics, Laboratory of Atomic and Solid State Physics, Cornell University, Ithaca, NY 14853-2501, USA 